\title{
An Innovative Hyperheuristic, Gaussian Clustering Scheme for Energy-Efficient Optimization in Wireless Sensor Networks
}

\author{
Oluwasegun Julius Aroba $\mathbb{D}^{1},{ }^{1}$ Nalindren Naicker $\mathbb{D}^{1},{ }^{1}$ and Timothy Adeliyi $\mathbb{D}^{2}$ \\ ${ }^{1}$ ICT and Society Research Group, Information Systems, Durban University of Technology, 4001 Durban, South Africa \\ ${ }^{2}$ ICT and Society Research Group, Information Technology, Durban University of Technology, 4001 Durban, South Africa \\ Correspondence should be addressed to Nalindren Naicker; nalindrenn@dut.ac.za
}

Received 25 November 2020; Revised 9 January 2021; Accepted 25 January 2021; Published 11 February 2021

Academic Editor: Carlos Marques

Copyright (C) 2021 Oluwasegun Julius Aroba et al. This is an open access article distributed under the Creative Commons Attribution License, which permits unrestricted use, distribution, and reproduction in any medium, provided the original work is properly cited.

\begin{abstract}
Energy stability on sensor nodes in wireless sensor networks (WSNs) is always an important challenge, especially during data capturing and transmission of packets. The recent advancement in distributed clustering algorithms in the extant literature proposed for energy efficiency showed refinements in deployment of sensor nodes, network duration stability, and throughput of information data that are channelled to the base station. However, much scope still exists for energy improvements in a heterogeneous WSN environment. This research study uses the Gaussian elimination method merged with distributed energy efficient clustering (referred to as DEEC-Gauss) to ensure energy efficient optimization in the wireless environment. The rationale behind the use of the novel DEEC-Gauss clustering algorithm is that it fills the gap in the literature as researchers have not been able to use this scheme before to carry out energy-efficient optimization in WSNs with 100 nodes, between 1,000 and 5000 rounds and still achieve a fast time output. In this study, using simulation, the performance of highly developed clustering algorithms, namely, DEEC, EDEEC_E, and DDEEC, was compared to the proposed Gaussian Elimination Clustering Algorithm (DEEC-Gauss). The results show that the proposed DEEC-Gauss Algorithm gives an average percentage of $4.2 \%$ improvement for the first node dead (FND), a further $2.8 \%$ improvement for the tenth node dead (TND), and the overall time of delivery was increased and optimized when compared with other contemporary algorithms.
\end{abstract}

\section{Introduction}

In this modern era, the use of the Internet of Things (IoT) gave birth to smart systems operating in wireless sensor networks (WSNs) that require little human interaction to carry out operations as it engages machine-to-machine communication $[1,2]$. The WSN system plays a crucial role in assembling information, and it is intended for remote area operation of applications where the physical appearance of humans is rare [3]. Wireless sensor networks have been explored in divergent sectors to display numerous monitoring obligations such as rescue, forage, target tracking, disaster recovery relief, health monitoring schedules, and several smart environmental activities. The location of sensors is important for routing algorithms [4]. These sensors collect data from a variety of sensor fields and distribute the information to the cloud server for analysis. Currently, the accuracy of locating the sensor nodes is the major issue in its applications [5]. The hierarchical-based model of routing is a process that ensures nodes with optimal power can be selected randomly for processing and sending information while low energy sensor nodes are used for sensing and sending data to the cluster heads. Clustering ensures that the wireless sensor network works in an effective form. Furthermore, it helps to speed up the energy consumption of sensors within the network lifetime [6].

Recent trends in technological growth have led to different advantages in modern day society; therefore, much scope exists for applied research on the energy efficiency of wireless sensor networks [7]. In WSNs, energy consumption is one of the major challenges as sensor nodes, and Cluster Heads have a short lifetime owing to demands in energy consumption [8, 9]. In recent times, different research works have focused on providing an effective WSN system for energy consumption, 
and many algorithms have been proposed to minimize GPS for the sensor node location identification. These include range free and range-based algorithms to estimate distance used and angles of connectivity for the information to travel between the known nodes and unknown sensor node locations $[10,11]$. WSNs are envisioned to be economic solutions in monitoring, home security, calculating of energy consumption within a time frame, and military surveillance. There has been a major problem with energy efficiency in WSN and its applications. Therefore, it is essential for the energy consumption to be used efficiently so that the network lifetime can be prolonged greatly.

The WSNs has a larger number of sensing devices which are usually called sensor nodes coupled with their battery life and range for sensors that are used in gathering data to the base station for coverage while the structured network needs to establish for feasibility in the coverage region such as streets, building, highways, and factories. These sensor nodes are interconnected, having its own cluster heads to pass communication through data which is sent to the base station (BS) for the information to be analyzed and processed within the heterogeneous network $[12,13]$. follows:

The primary objectives of this research work are as

(i) To provide an effective scheme for optimizing energy efficiency.

(ii) To perform an extensive simulation of the proposed DEEC-Gauss algorithm in MATLAB

(iii) To analyze and evaluate the performance of the proposed algorithm with cutting edge algorithms based on well-known metrics such as first node dead (FND), tenth node dead (TND), network lifetime, and packets of data sent to the base station.

The research paper is organized as follows: the literature review is explicated in Section II. We further described in Section III the proposed material and methods for the DEEC-Gauss novel algorithm for optimization of energy efficiency consumption. Section IV explained the simulation experiment and analysis of the proposed algorithm compared with three other state-of-the-art algorithms. Furthermore, performance metrics such as first node dead (FND), tenth node dead (TND), packets delivered to the base station, and processing time were used to ascertain the effectiveness and efficiency of the proposed algorithm. Finally, Section V presents the conclusion and future recommendation scope.

\section{Literature Review}

There are several energy efficiency approaches reported in the literature on node localization. Hyperheuristic schemes are high level approaches with combinatorial optimization resolutions [14-18]. Hyperheuristics are used to resolve hard computational search problems. A systematic review and meta-analysis revealed that studies that used the hyperheuristic approach gave the best result in optimizing the node localization and energy efficiency problem, hence increasing the WSN performance [19]. However, it does not mean that metaheuristic and hybrid heuristic are not efficient, but the analysis proved that the hyperheuristic performance outperforms the metaheuristic and hybrid heuristic approaches in optimizing the node localization and energy efficiency problems in WSN [20]. Data processing fusion methods are classified as probabilistic, knowledge-based, and reasoning methods for data aggregation and statistical methods that have been used in recent years for feature preservation of data outlier detection $[21,22]$.

The Differential Evolution (DE) algorithm and the Particle Swarm Optimization (PSO) algorithm were, separately, explored to solve the clustering problem in wireless sensor networks $[23,24]$. Their focus was on maximization of coverage and prolonging the network lifetime in WSNs. Research by Cui. et al. in [25], suggested that the DE algorithm was presented for the situation in which the communication range value was unknown to deal with the optimization problem. Cespedes-Mota et al. in [26] utilised the multiobjective DE algorithm to improve the sensor distribution over the multiple areas within the geolocation and to expand the coverage area and to minimize the network energy consumption at the same time. The PSO algorithm is a nature inspired algorithm by the social behavior of birds, has been one of the most popular optimization algorithms that is widely applied to solve complex optimization problems in WSNs. Combining PSO with a random algorithm for transition moves was proposed as an hybrid approach to create the maximum possible number of disjoint sets to increase the lifetime of WSNs [27]. In another words, for the path optimization problem in WSNs, the PSO algorithm was applied for energy consumption and idle listening energy consumption [28].

Several studies suggested that a self-organizing network clustering method based on a genetic algorithm (GA) provided the most advantageous network organization to expand the network lifetime in WSNs [29]. Yan et.al in [28] suggested the GA-based modified LEACH algorithm for energy harvesting in WSN (EH-WSNs) for minimizing energy use and increase the network lifetime of EH-WSNs. Over time, extensive research work by Yuan et al. in [29] proposed a self-organizing network clustering, GASONeC method, to boost the network lifetime. Wang et al. in [30] emphasized that a distributed GA solved energy-efficient coverage problems in WSNs. Hamidouche in [31] employed another method of distributed GA to solve energy-efficient coverage problems in WSNs to prolong the quality of service and expand network lifetime.

GA-based approaches for clustering and routing in WSNs to prolong the network lifetime of sensors and to speed up the quality of service were proposed in the extant literature [32]. Seminal contributions have been made by Singh and Sharma in [33], who utilised the PSO algorithm to lower the location error and advance location accuracy in WSNs. Hence, there is a possible increase in the lifetime of the network. Singh and Sharma in [33] with help of the PSO algorithm examined a novel energy-efficient clustering algorithm for WSNs that improves the life span of the wireless sensor network. The most focused objective 
of the proposed algorithm was the selection of a corresponding cluster head, to minimize the intracluster distance and increase the energy efficiency of the network. The authors proposed the method which uses a high powered node as cluster head and generated the clusters by placing them in a consistent pattern in the sensor region. The authors attempted to maximize data transmission whilst at the same time optimize energy consumption by the nodes.

Load balance is a way to coordinate energy usage and the rate of usage. Singh and Sharma in [33] suggested that load balancing does not equate to the similar distribution of network loads but display how the load is balanced on the single node according to the status of the network operation. Another method to balance the load is through the sensor clustering mechanism. Clusters come together to form a cluster of networks. In the extant literature on WSNs data-driven approaches, duty cycling, and various mobility-based approaches are used for energy consumption. Wireless sensor networks have sensor devices to use in ad-hoc networks and battery-operated computing mechanism [34]. It stands to reason that if energy consumption is increased then network lifetime would also be optimized. In cluster-based WSNs, a cluster gateway performs behaviors like data collection from their member nodes, data exchange, and data aggregation to the base station destination. The load balancing of cluster head to be a crucial parameter in the optimization of the lifetime in WSNs is one of the key components. The clustering algorithm LEACH applicable for wireless sensor networks is not controlled by any central point. This distributed algorithm involved in clustering the LEACH leaves the nodes independent. In addition, there are some algorithms that are within the jurisdiction of LEACH which are hierarchical routing which are considered as one of the most preferable algorithms. Local cluster heads $(\mathrm{CHs})$ carry out the construction of a cluster of nodes. The different clusters serve as router that mediate between the sink node that help to channel the signal strength. CHs carry out the task of data transmission rather than every other node in the network. After the selection of transmission via. the routing, energy is being optimized and saved by retaining the temporal data processing. If there is an alternative change in the $\mathrm{CH}$ at a randomize level, the balance of dissipation of node will be made possible for the energy sensor nodes. The $\mathrm{CHs}$ carry out this task by making sure there is stability with the use of random sequence between 1 and 0 . The individual nodes are presented with a $\mathrm{CH}$ for the real time round to carry out the task of checking if their number is not lesser than the threshold quota [35].

LEACH has a distinct disadvantage in that it is not feasible for functioning in networks of a wider environment. This is because the cluster heads in the LEACH process affect communication with the distant base station, thereby consuming more power for data transmission. Furthermore, the ultimate effect of this is the reduced lifetime of the network. However, LEACH has the feasibility of making the randomized rotation of the cluster head throughout the network. In this way, less energy is consumed for transmission when LEACH chooses the specific cluster head. In other words, the $\mathrm{CHs}$ might have different initial energy requirements which is referred to as a heterogeneous network or the same initial energy requirements which is referred to as a homogenous network [36, 37].

In the light of reported literature, Kianiand and Seyyedabbas in [38] proposed the routing, scheduling, channel assignment, and power control problems in multipower level for WSNs as an integer linear programming effect. This led to the blueprint of distributed routing protocols which help to reduce the rate of energy consumption. The modified time division multiple access (TDMA) was enhanced for the cluster head selection method to improve the scheduling approach for reducing energy dissipation for sensor nodes with residual energy consumption [39,40]. Seminal research conducted by Roselin et al. in [41] employed the novel approach of energy efficiency to connect coverage scheduling lifetime of WSNs.

The advantages of swarm intelligence is that it ensures adaptability and stability of the environment which is useful when needed to conserve energy in wireless sensor networks [42, 43]. Furthermore, the bioinspired PSO has become one of the most significant optimization algorithms and has been extensively utilised to solve complex optimization problems. Pang et al. in [44] and Wang et al. in [45] applied the PSO algorithm to lower the location error and enhance the accuracy of the location to avoid energy holes near the cluster caused by the heavy burden for forwarding data packets in WSNs. Similarly, WSNs have got considerable attention from various sides. Wang et al. in [45] presented a novel secret confusionbased energy-saving and privacy-preserving data aggregation algorithm to reduce data traffic and energy usage in WSNs.

It was inevitable in a modern day developed lifestyle to solve the emerged problem of energy efficiency to ensure effective services. Therefore, different algorithms have been proposed in the extant literature to deal with the energyefficient problem to reduce energy consumption in operational methods of WSNs [46]. The next section discusses the materials and methods used in this research study.

\section{Materials and Methods}

3.1. DEEC-Gauss Network Method Model. In this section, we introduce the novel DEEC-Gaussian algorithm model which helps the network comprise of energy-efficient clustering with distance between the cluster head $(\mathrm{CH})$ and sensor nodes. The $P_{\text {opt }}=0.1$ is one of the parameters used for the period mode during the DEEC-Gauss algorithm. We employ and deploy all the sensor nodes randomly and those that are stationary at one point. These various individual nodes comprise of the same limited amount of energy beginning from $0.5 \mathrm{~J}$ to $0.8 \mathrm{~J}$. The base station (BS) is a homogeneous station which is within and outside the sensing environment. These sensor nodes are aggregated between a time interval periodically, which is then transferred to the cluster head. The sensor node location is not known nor the position in which they are situated. 
Consequently, the self-organizing is being monitored before deployment to the next operation.

The homogeneous nodes can operate as a cluster head, and the nodes are then sent within the same time at random to be able to ascertain the network lifetime of the sensor node with the expected energy outcome after the task is completed at each round. The Cluster head $(\mathrm{CH})$ is situated at the centre of the sensing area. There is a drastic reduction in the number of sensor nodes that are deployed from the base station which is the desired destination of all data packets. Before the data aggregated arrival, the nodes do travel to the $\mathrm{CH}$ which makes it difficult for the cluster head $(\mathrm{CH})$ to determine their locations.

This simulation above was done in MATLAB R2020a and their properties have some limitations for the node to compute the distance between the base station and the node by differentiating the received signal strength. It does not require any additional systems with location services to determine the GPS. Hence, the various nodes collaborate with the CHs that is the closest to it.

\subsection{Proposed Heterogeneous Optimized DEEC-Gauss Energy} Algorithm. This energy model helps to identify the energy transmitted when the nodes acknowledge or transfer data within the network. In this paper, we adopted the DEEC state-of-the-art algorithm and further improved on the work with a clustering-based algorithm that is titled Gaussian Elimination and its criteria formulations. The proposed DEEC-Gauss Algorithm 1 helps to provide an efficient solution as highlighted below with the use of the pseudocode procedure below:

Step 1. We set the parameter model.

Step 2. We initialize the energy parameters for all sensor nodes with the use of the formula below [47]:

$$
E_{\text {Total }}=\sum_{i=1}^{n} E_{0}\left(1+a_{i}\right)=E_{0}\left(\left(n+\sum_{i=1}^{n} a_{i}\right)\right) .
$$

Step 3. Start the process of iteration of the rounds as follows:

(i) See if any individual dead node exists and note the round the first node is dead

(j) See if $10 \%$ of the nodes are dead and save the round this occurs

(k) See if all the nodes are dead and save the round the next process to occurs

(1) Check each node if alive and label as "N"

(m) Save the number of dead nodes, alive nodes, and cluster heads which are set at initial zero in every round

(n) Iterate through each node

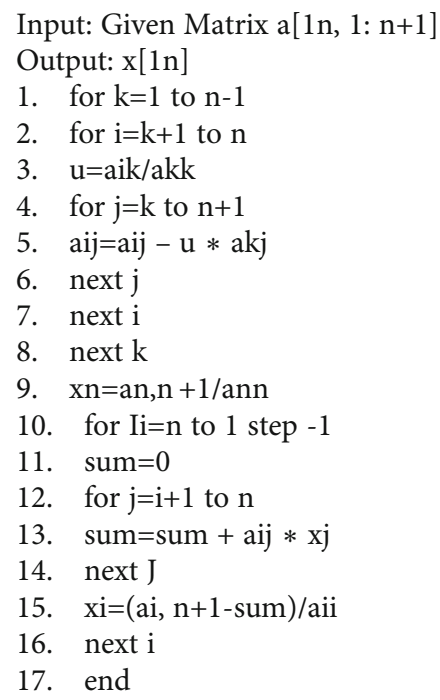

Algorithm 1: Proposed Gauss Elimination Algorithm [49].

(o) Calculate the $p_{i}$ for heterogeneous nodes using [48]:

$$
p_{i}=\frac{p_{\mathrm{opt}} N(1+a) E_{i}(r)}{\left(N+\sum_{i=1}^{N} a_{i}\right) \bar{E}(r)}
$$

(p) Calculate the energy required by the transmit amplifier [48]

$$
E_{\mathrm{TX}}(l, d)=\left\{\begin{array}{ll}
l E_{\mathrm{elec}}+l \varepsilon_{f_{s}} d^{2}, & d<d_{0} \\
l E_{\mathrm{elec}}+l \varepsilon_{\mathrm{mp}} d^{4}, & d \geq d_{0}
\end{array}\right\}
$$

and compute the energy needed by the receiver $E_{\mathrm{RX}}$ (l.) $[48]$

$$
\text { using } E_{\mathrm{RX}}(l)=l E_{\text {elec }}
$$

(q) Calculate the energy used for the sensor node in the next iteration [49]

$$
E_{\text {Total }}=\sum_{i=1}^{n} E_{0}\left(1+a_{i}\right)=E_{0}\left(\left(n+\sum_{i=1}^{n} a_{i}\right)\right)
$$

Let $n_{i}$ represent the number of rounds to be the individual cluster head for the sensor node $s_{i}$, and we call it the rotating epoch, super node, and normal node. In the recognised DEEC method, $E_{i}$ is used for energy dissipated in the network. In our proposed scheme, $E_{i}$ is used to represent the residual energy $E_{i}$ ${ }^{\circledR}$ of the node $s_{i}$ at round $r$. When the cluster heads have been chosen using DEEC, the selected cluster heads are now subjected to the Gaussian elimination algorithm.

We assume that the number of selected cluster heads is q; matrix $A$ represents the energy consumption of every node 
chosen as cluster head and $q$ the number of cluster heads. $a_{i j}$ represent the energy utilised by a cluster head $i$ taken to be normal node if cluster head $j$ is its cluster head. Furthermore, $b_{i}$ represents the residual power of cluster head $i$, while $x_{i}$ expresses the times that cluster head $i$ will become a cluster head. In this way, matrices $B$ and $X$ are established so that $A X=B$, as shown in Equation (6) below [49]:

$$
\left[\begin{array}{ccccc}
a_{11} & a_{12} & a_{13} & & a_{1 k} \\
a_{21} & a_{22} & a_{23} & \cdots & a_{2 k} \\
a_{31} & a_{32} & a_{33} & & a_{3 k} \\
& \vdots & & \ddots & \vdots \\
a_{k 1} & a_{k 2} a_{k 3} & \cdots & a_{k k}
\end{array}\right]\left[\begin{array}{c}
x_{1} \\
x_{2} \\
x_{3} \\
\vdots \\
x_{k}
\end{array}\right]=\left[\begin{array}{c}
b_{1} \\
b_{2} \\
b_{3} \\
\vdots \\
b_{k}
\end{array}\right] .
$$

The piece of code below is used to calculate number of rounds within the network to obtain optimal number of clusters $[49,50]$.

For $(k=1 ; k<m+1 ; k++)$

$I_{-} \max :=\operatorname{argmax}(i=k \cdots m, \operatorname{abs}(A[i, k]))$;

If $\left(A\left[i \_\max , k\right]=0\right)$

Error "Matrix is singular!";

Swap rows ( $k$,i_max).

The piece of code below is used to calculate the number of packets sent to the base station and the tenth node dead [49].

For $(i=k+1 ; i<m+1 ; i++)$

For $(j=k+1 ; k<n+1 ; j++)$

$A[i, j]:=A[i, j]-A[k, j] x(A[i, k] / A[k, k])$;

$A[i, k]:=0$;

Figure 1 shows the system model of the proposed DEECGaussian algorithm.

3.3. Energy Consumption. The energy usage varies from $0.5 \mathrm{~J}$ and $0.6 \mathrm{~J}$ to $0.7 \mathrm{~J}$ and $0.8 \mathrm{~J}$ initiated at every point for the sensors by clustering nodes of the sensors in the network. The proposed approach with the use of the DEEC-Gauss algorithm helps to provide an adaptive efficient use of energy resources of sensor nodes.

3.4. Performance Evaluation. The proposed DEEC-Gauss was simulated in MATLAB 2020b, and the results were plotted. The system configuration was Intel Core i7-8650U CPU @1.90GHz, 2.11 GHZ, installed memory (RAM) 8,00GB (7,85 GB usable), System type 64-bit operating system, the x64-based processor running Windows 10 . The simulation was performed many times with varying conditions such as the number of nodes and the number of cluster heads. The network sensing area was pivoted to $100 \times 100 \mathrm{~m}^{2}$. The initial simulations were on WSN \#1 with 100 nodes and 10 cluster heads. The base station was positioned at the edge of the sensing area at $(100 \times 100)$. The DEEC-Gauss, DEEC-E, DDEEC_E, and DEEC were run 120 times, and the average of the instances of the resultant data was selected for plotting results. The DEEC-Gauss algorithm was tested with a predefined clustering population. The various parameters considered for the simulation are given in Table 1 below.

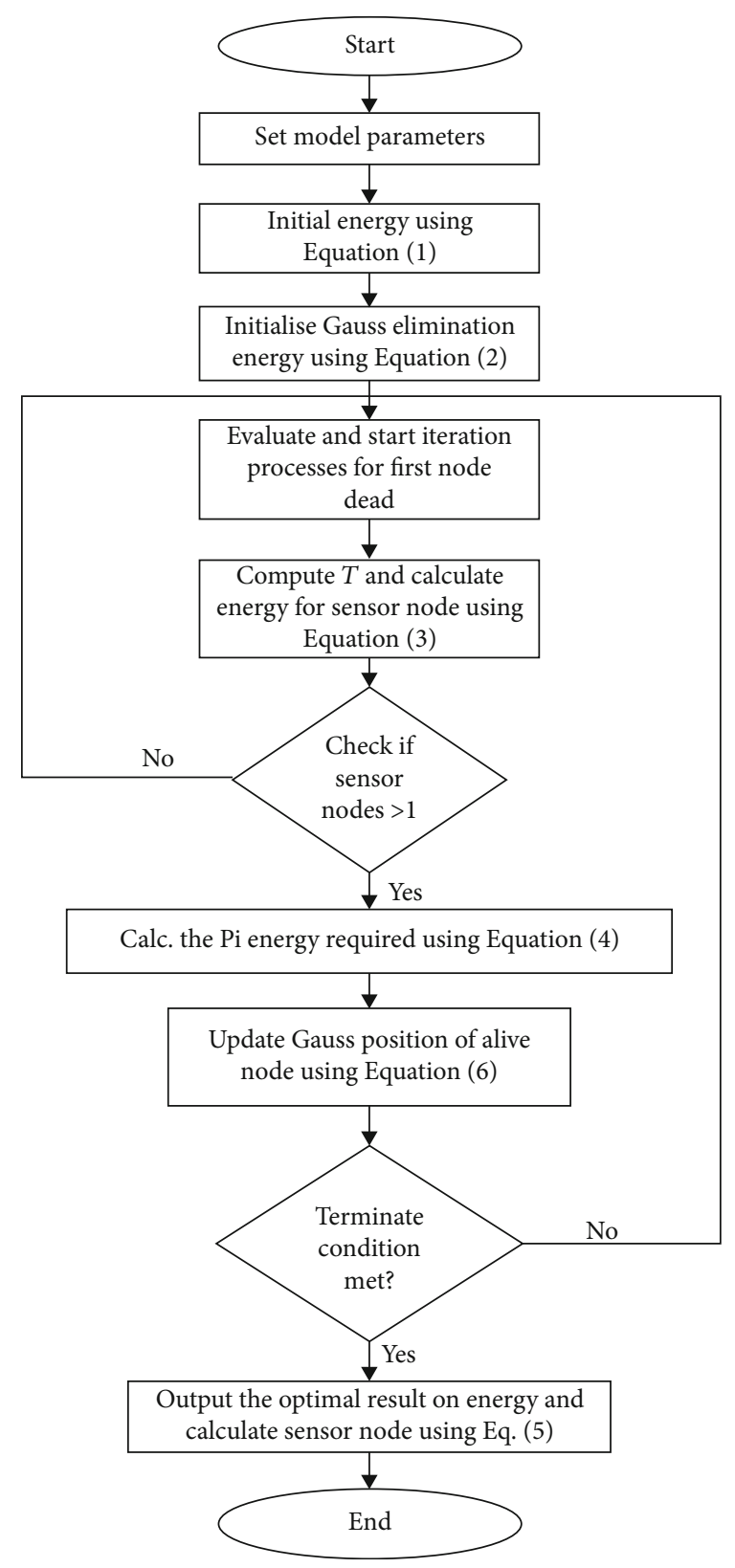

FIgURE 1: System model of the proposed DEEC-Gaussian algorithm.

3.5. The Performance Criteria Used. The metric of parameters used for the performance and evaluation of the clustering operation protocols are lifetime, the number of sensor nodes, alive nodes, and number of data packets received at the base station.

(i) Data packet acknowledged at the base station is the total number of data packets of information that are acknowledged at the destination (base station), and their output is different from time to time.

(ii) Alive Nodes is the measurement of the total number of nodes that has not used up their energy. 
TABLE 1: Simulation network parameters.

\begin{tabular}{lc}
\hline Parameters & Value \\
\hline Network field & $(100,100) \mathrm{m}^{2}$ \\
Number of nodes & 100 \\
Eo (initial energy of normal nodes) & $0.5 \mathrm{~J}-0.8 \mathrm{~J}$ \\
Message size & $4000 \mathrm{bits}$ \\
$E_{\text {elec }}$ & $50 \mathrm{~nJ} / \mathrm{bit}$ \\
$E_{\mathrm{fs}}$ & $10 \mathrm{~nJ} / \mathrm{bit} / \mathrm{m}^{2}$ \\
$E_{\text {amp }}$ & $0.0013 \mathrm{pJ} / \mathrm{bit} / \mathrm{m}^{4}$ \\
EDA & $5 \mathrm{~nJ} / \mathrm{bit} / \mathrm{signal}$ \\
$D_{o}$ (threshold distance) & $100 \mathrm{~m}$ \\
$P_{\text {opt }}$ & 0.1 \\
\hline
\end{tabular}

Table 2: Alive nodes during the network lifetime for 100 nodes using the initial energy at $0.5 \mathrm{~J}$.

\begin{tabular}{lccccc}
\hline Algorithms & $P_{\text {opt }}$ & FND & $\begin{array}{c}\text { Tenth node } \\
\text { dead }\end{array}$ & $\begin{array}{c}\text { Packet to } \\
\text { BS }\end{array}$ & Time (S) \\
\hline DEEC_E & 0.1 & 1444 & 1693 & 82268 & 8,17027 \\
DDEEC_E & 0.1 & 1344 & 1440 & 89892 & 8,727181 \\
DEEC & 0.1 & 1282 & 1552 & 136305 & 9,984828 \\
DEEC_ & 0.1 & 1617 & 1832 & 133944 & 5,088432 \\
GAUS & & & & & \\
\hline
\end{tabular}

TABle 3: Alive nodes during the network lifetime for 100 nodes using the initial $0.6 \mathrm{~J}$.

\begin{tabular}{lccccc}
\hline Algorithms & $P_{\text {opt }}$ & FND & $\begin{array}{c}\text { Tenth node } \\
\text { dead }\end{array}$ & $\begin{array}{c}\text { Packet to } \\
\text { BS }\end{array}$ & Time (S) \\
\hline DEEC_E & 0.1 & 1308 & 1514 & 89892 & 8,727181 \\
DDEEC_E & 0.1 & 1451 & 1719 & 112310 & 9,210859 \\
DEEC & 0.1 & 1768 & 2114 & 138482 & 9,187751 \\
DEEC_ & 0.1 & 2064 & 2236 & 159276 & 6,719211 \\
GAUS & & & & & \\
\hline
\end{tabular}

\section{Simulation and Analysis}

In this segment, we present the simulation results of the proposed novel DEEC-Gauss algorithm and the comparison analysis with well recognised clustering algorithms, namely, Developed Distributed Energy-Efficient Clustering Extended (DEEC_E), Developed Distributed Energy-Efficient Clustering Extends (DDEEC_E), and Distributed Energy-Efficient Clustering (DEEC) with normal, advanced, and super node classifications.

A clustering algorithm was evaluated in terms of stability of the network, namely, the number of rounds in the network until the first node dies (FND) and the number of rounds in the network until the tenth node dies, and their energy gets depreciated, represented as tenth node dead (TND) in the tables below. Tables 2-5 below are the output of the simulation model for DEEC_E: Developed Energy-Efficient Clustering Extended, DDEEC_E: Developed Distributed Energy-Efficient Clustering Extended, DEEC: Distributed
TABLE 4: Alive nodes during network lifetime for 100 nodes (initial energy of $0.7 \mathrm{~J}$ ).

\begin{tabular}{lccccc}
\hline Algorithms & $P_{\text {opt }}$ & FND & $\begin{array}{c}\text { Tenth node } \\
\text { dead }\end{array}$ & $\begin{array}{c}\text { Packet to } \\
\text { BS }\end{array}$ & Time (S) \\
\hline DEEC_E & 0.1 & 1484 & 1918 & 174625 & 7,66522 \\
DDEEC_E & 0.1 & 1827 & 2023 & 152256 & 9,187751 \\
DEEC & 0.1 & 1585 & 1868 & 110453 & 11,17691 \\
DEEC- & 0.1 & 2028 & 2292 & 185463 & 7,794244 \\
Gaussian & & & & & \\
\hline
\end{tabular}

TABLE 5: Alive nodes during network lifetime for 100 nodes (initial energy of $0.8 \mathrm{~J}$ ).

\begin{tabular}{lccccc}
\hline Algorithms & $P_{\text {opt }}$ & FND & $\begin{array}{c}\text { Tenth node } \\
\text { dead }\end{array}$ & $\begin{array}{c}\text { Packet to } \\
\text { BS }\end{array}$ & Time (S) \\
\hline DEEC_E & 0.1 & 2190 & 1693 & 82268 & 7,260549 \\
DDEEC_E & 0.1 & 2005 & 2370 & 171984 & 10,27297 \\
DEEC & 0.1 & 1894 & 2150 & 142979 & 10,37544 \\
DEEC- & 0.1 & 2480 & 2751 & 213305 & 10,43691 \\
Gaussian & & & & & \\
\hline
\end{tabular}

Energy-Efficient Clustering, and DEEC_GAUS: Distributed Energy-Efficient Clustering Gaussian Elimination Clustering algorithms. In Tables 2-5, the acronyms BS and S refer to the base station and time taken to complete in seconds, respectively.

Tables 2-5 below show the results of the proposed DEEC-Gauss algorithm and the state-of-the-art algorithms, namely, DEEC_E, DDEEC_E, and DEEC for heterogeneous initial energy values of between $0.5 \mathrm{~J}$ and $0.8 \mathrm{~J}$.

Table 2 shows that the novel DEEC-Gauss algorithm had the fastest time to run for the operation of sensor nodes with initial energy of $0.5 \mathrm{~J}$. The number of packets sent to BS (133944) was more on the DEEC_GAUS than state-of-theart clustering algorithms. When the first node dies, performance tends to decline, and the network becomes unstable. The proposed DEEC_GAUS produced the best results for FND and tenth node dead. The FND was reported at round 1444, and tenth node dead was reported at round 1693.

Table 3 presents the results of 100 nodes using initial energy of $0.6 \mathrm{~J}$.

Table 3 shows that the number of packets sent to BS is more on the proposed DEEC_GAUS (159276) than stateof-the-art clustering algorithms, namely, DEEC_E, DDEEC_E, and DEEC. The proposed DEEC_GAUS produced the best results for FND (2064 rounds) and tenth node dead (2236 rounds).

Table 4 presents the results 100 nodes using initial energy of $0.7 \mathrm{~J}$.

Table 4 shows that the number of packets sent to BS is more on the DEEC GAUS (185463) than state-of-the-art clustering algorithms, namely, DEEC_E, DDEEC_E, and DEEC. The proposed DEEC_GAUS produced the best results for FND (2028 rounds) and tenth node dead (2292 rounds).

Table 5 presents the results 100 nodes using initial energy of $0.8 \mathrm{~J}$. 


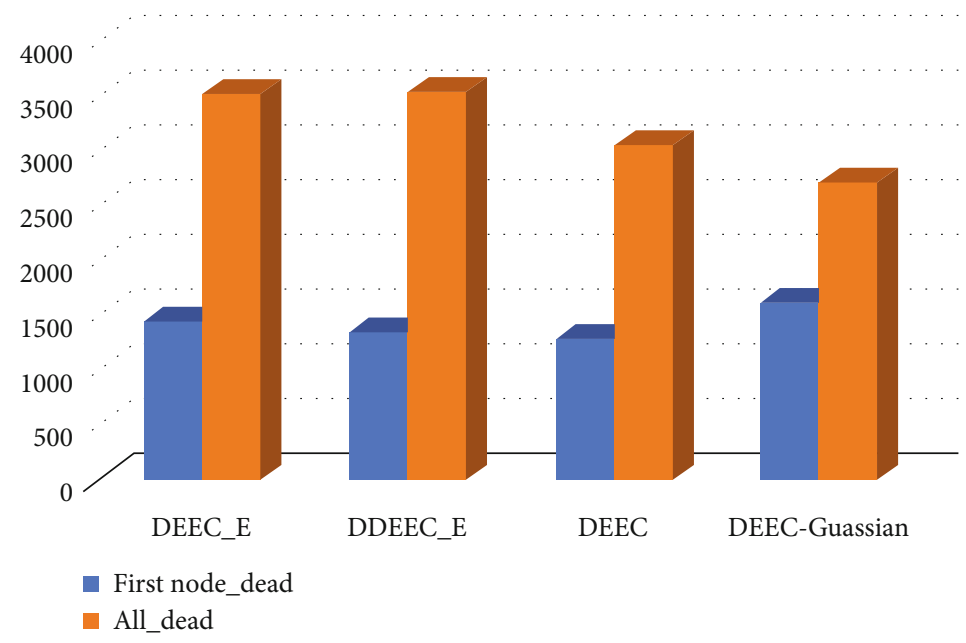

FIGURE 2: First node_dead and all_dead node for $0.5 \mathrm{~J}$.

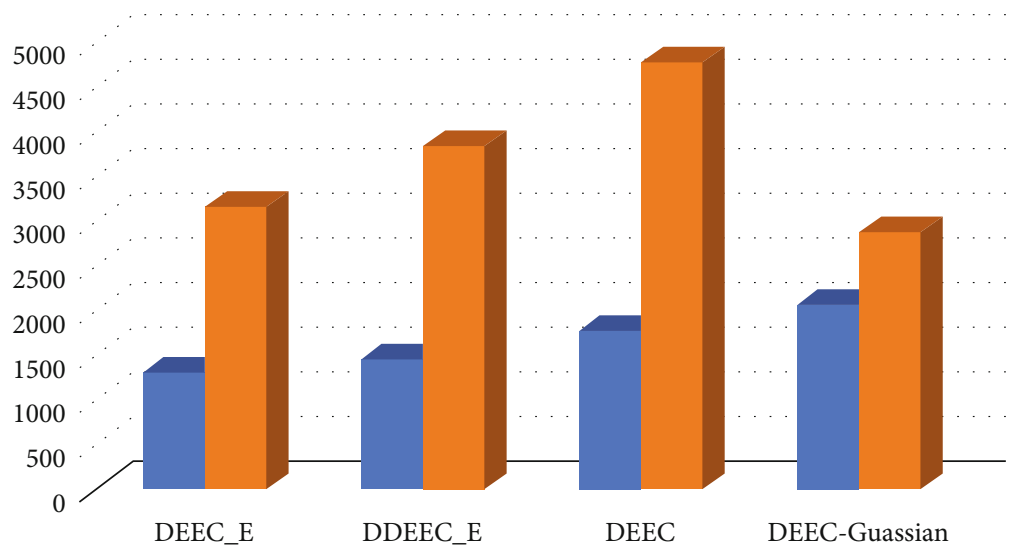

- First node_dead

- All_dead

Figure 3: First node_dead and all_dead node for 0.6 J.

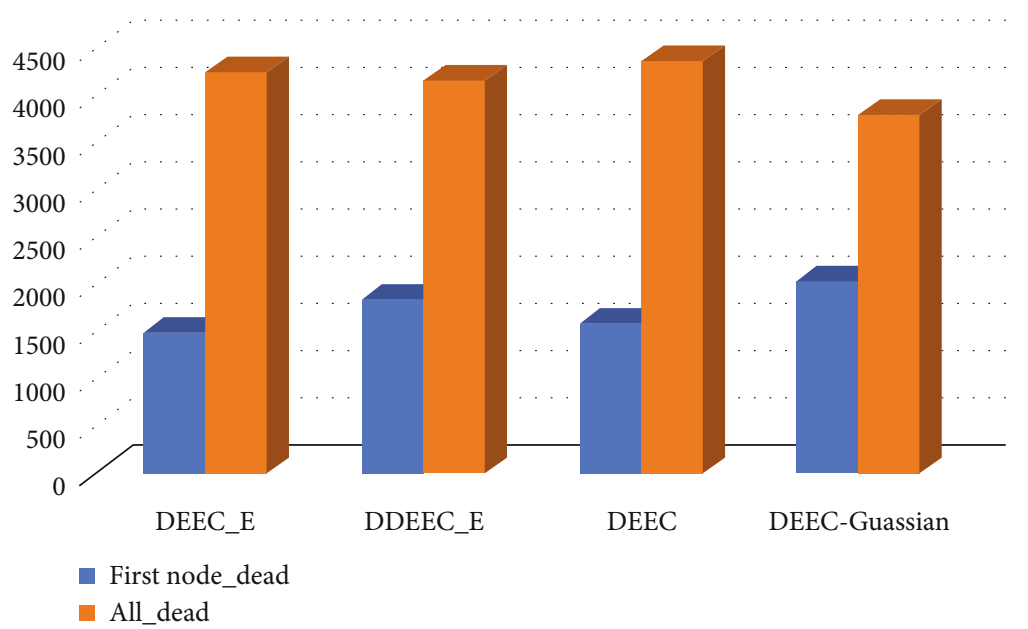

FIGURE 4: First node_dead and all_dead node for $0.7 \mathrm{~J}$.

Table 5 shows that the number of packets sent to BS is more on the DEEC_GAUS (213305) than state-of-the-art clustering algorithms, namely, DEEC_E, DDEEC_E, and
DEEC. The novel DEEC_GAUS also has the advantage of producing the best results of 2480 rounds for FND and 2751 rounds for the tenth node dead for $0.8 \mathrm{~J}$ of initial energy. 


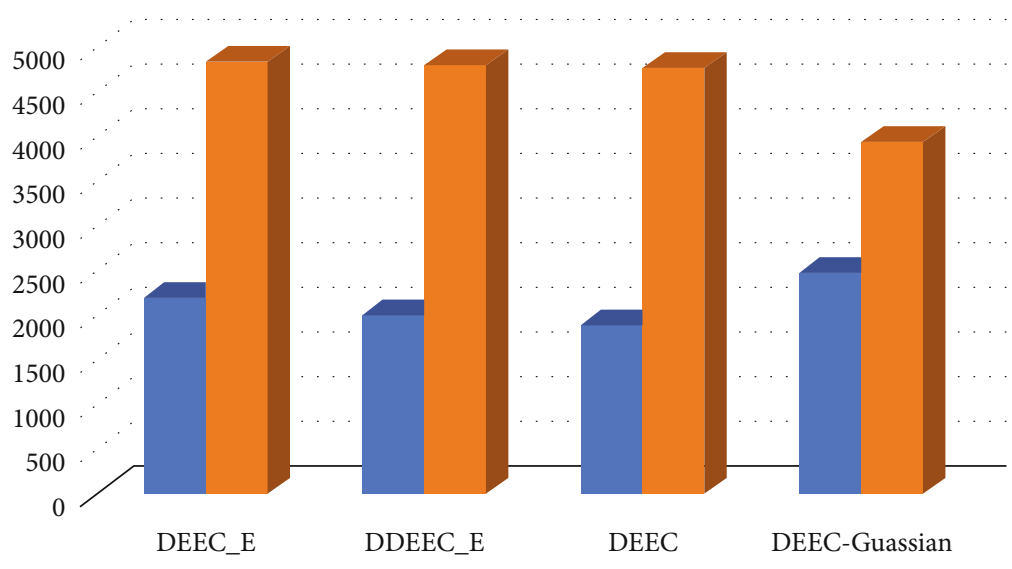

- First node_dead

- All_dead

FIgURE 5: First node dead and all_dead node for $0.8 \mathrm{~J}$.

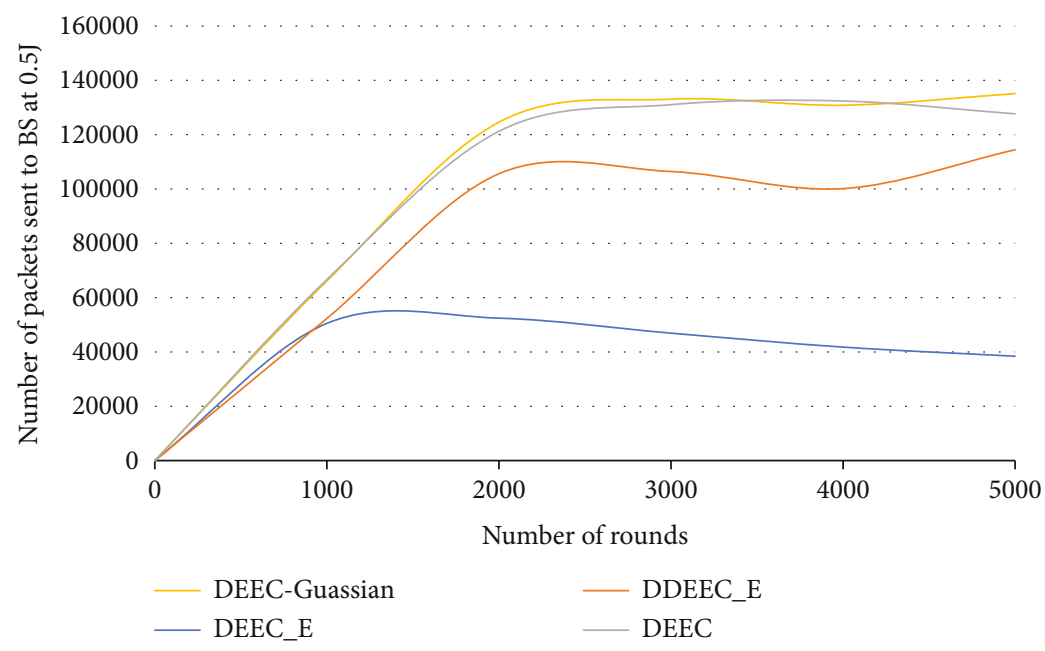

Figure 6: Number of packets sent to the base station vs number of rounds for $0.5 \mathrm{~J}$.

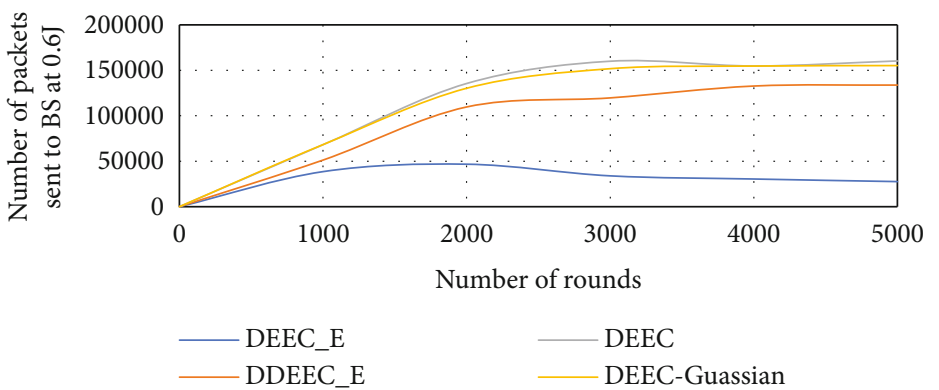

Figure 7: Number of packets sent to the base station vs number of rounds for $0.6 \mathrm{~J}$.

4.1. Comparative Analysis of First Node Dead (FND) and All Dead Nodes. The result of the proposed DEEC_GAUS from Tables 2-5 was able to establish a stable period before it throws up the first dead node, and the lifetime of the network performance tends to reduce. The displayed results were presented in Tables 2-5 for heterogeneous initial energy that was consumed for the range of $0.5 \mathrm{~J}, 0.6 \mathrm{~J}, 0.7 \mathrm{~J}$, and $0.8 \mathrm{~J}$. The performance of the FND for DEEC-Gauss, DEEC_E,
DDEEC_E, DEEC, and the novel DEEC_GAUS for the different energy levels can be observed in Tables 2-5. On the other hand, it is deduced that the FND of DEEC_E, DDEEC_E, and DEEC is inferior to the proposed DEEC_GAUS as shown in Figures 1-4.

Similarly, we observed the performance of the proposed DEEC_GAUS algorithm in selecting the optimal cluster head, and the same $0.1 P_{\text {opt }}$ parameters were used all through 


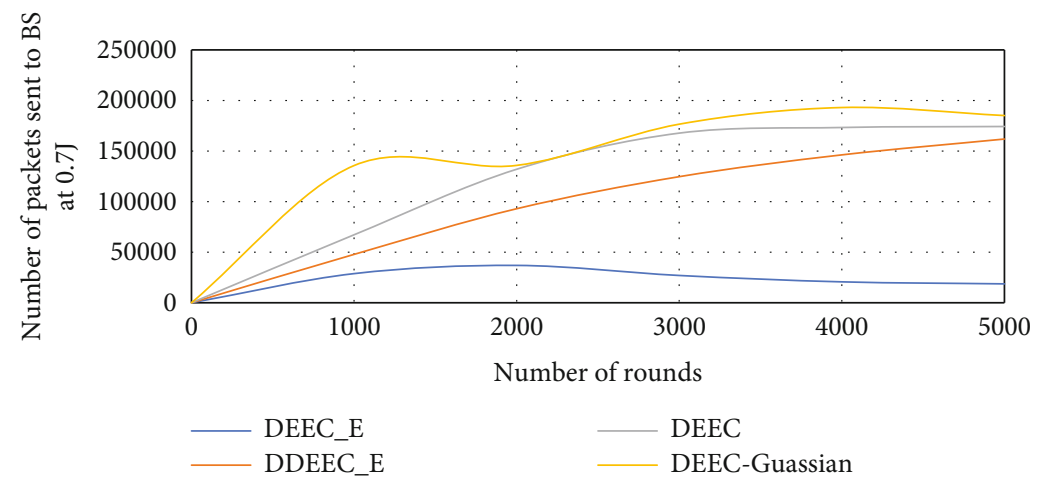

Figure 8: Number of packets sent to the base station vs number of rounds for $0.7 \mathrm{~J}$.

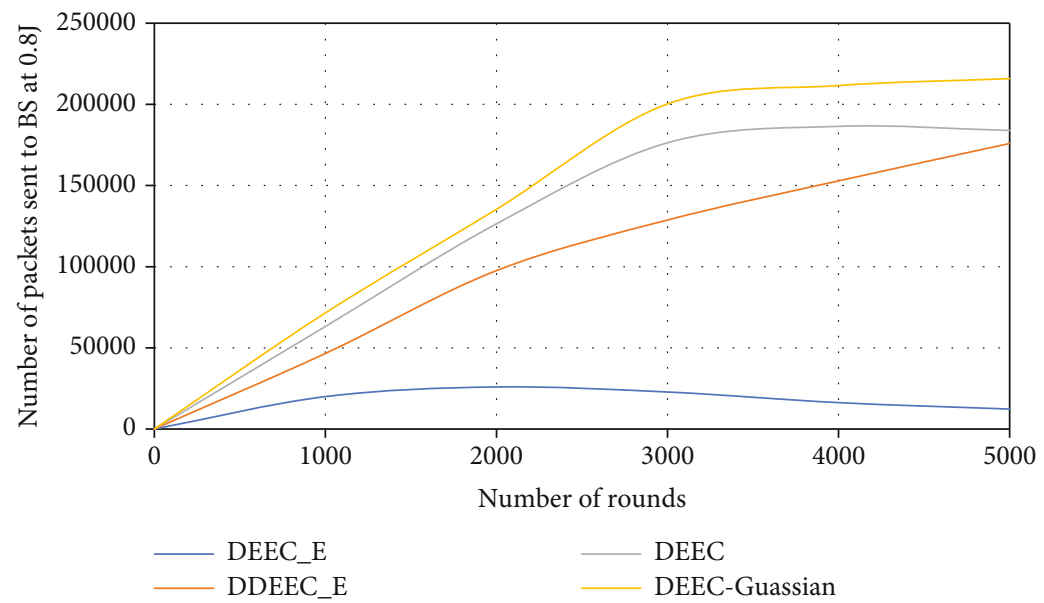

Figure 9: Number of Packets sent to Base Station vs Number of rounds for 0.6 J.

the program that was generated randomly. However, the network performance represented on the bar chart displayed the clarity of the algorithm compared to our proposed novel DEEC_GAUS clustering algorithm, for FND, all dead node, and initial energy for $0.5 \mathrm{~J}, 0.6 \mathrm{~J}, 0.7 \mathrm{~J}$, and $0.8 \mathrm{~J}$.

We can draw our conclusion from the simulation and analysis shown from Figure 2 to Figure 5 with the initial energy from $0.5 \mathrm{~J}$ to $0.8 \mathrm{~J}$, the comparison for the first node, tenth node, the times it takes for each round to run, and we can ascertain that our proposed DEEC_GAUS algorithm has done well in all, respectively.

Figure 2 presents the results for the first node dead and all dead nodes for DEEC_E, DDEEC_E, DEEC, and the novel DEEC_GAUS.

The Figure above shows that DEEC_GAUS produced the best results for the first node dead and all dead node for $0.5 \mathrm{~J}$ of energy.

Figure 3 represents the results for the first node dead and all dead nodes for DEEC_E, DDEEC_E, DEEC, and the novel DEEC_GAUS.

The figure above shows that DEEC_GAUS produced the best results for first node dead and all dead node for $0.6 \mathrm{~J}$ of energy.

Figure 4 shows the results for the first node dead and all dead nodes for DEEC_E, DDEEC_E, DEEC, and the novel DEEC_GAUS
The figure above shows that DEEC_GAUS produced the best results for first node dead, and DEEC produced the best results for all dead nodes for $0.7 \mathrm{~J}$ of energy.

Figure 5 shows the results for the first node dead and all dead nodes for DEEC_E, DDEEC_E, DEEC, and the novel DEEC_GAUS.

The figure above shows DEEC_GAUS produced the best results for first node dead, and DDEEC_E produced the best results for all dead nodes for $0.7 \mathrm{~J}$ of energy.

4.2. The Comparative Analysis of Network Throughput. The network throughput is one of the foundational requirements in WSN to assess the performance of the various algorithms. They are many data packets that are transferred within the network successfully to the base station (destination). The packets from the $\mathrm{CH}$ are sent to the base station and during this period of operation. The $\mathrm{CH}$ gets to fuse the data received and dispatch it to the base station and at this time the energy of the Cluster Head must be sufficient to accept or send packets.

This simulation is performed with the 4 categories of algorithms DEEC_E, DDEEC_E, DEEC, and the novel DEEC_GAUS using 100 nodes with various heterogeneous initial energies between 0.5 and $0.8 \mathrm{~J}$. Figures $6-9$ show the number of packets sent to the base station as the number of 
rounds increase. A graph of packets to BS vs number of rounds is plotted below.

Figure 6 shows the performance of algorithms for $0.5 \mathrm{~J}$ of initial energy, and the proposed DEEC_GAUS shows the highest network throughput of in excess of 12000 packets in 5000 rounds.

The figure below shows the number of packets sent to the base station vs number of rounds for $0.6 \mathrm{~J}$ of energy.

Figure 7 shows the performance of algorithms for $0.6 \mathrm{~J}$ of initial energy, and the proposed DEEC_GAUS shows the highest network throughput of in excess of 15000 packets in 5000 rounds.

The figure below shows the number of packets sent to the base station vs number of rounds for $0.7 \mathrm{~J}$ of energy.

Figure 8 shows the performance of algorithms for $0.7 \mathrm{~J}$ of initial energy, and the proposed DEEC_GAUS shows the highest network throughput close to 20000 packets in 5000 rounds

The figure below shows the number of packets sent to the base station vs Number of rounds for $0.8 \mathrm{~J}$ of energy.

Figures 6-9 show the number of packets sent successfully to the base station with the novel DEEC_GAUS that produced the highest network throughput in all the cases of energy of the simulation results. The outcome of the simulation overall shows that DEEC_GAUS is the most efficient algorithm since it sends many packets to the BS. Similarly, it is the best efficient clustering algorithm to dispatch a higher number of packets that have low energy consumption in the WSN. It has shown to have a low consumption of energy and has the best network stability duration. Thus, it is evidenced in the network lifetime and in the number of rounds.

\section{Conclusions}

This research paper presented a novel DEEC_GAUSS approach for the optimization of localization and energy efficiency in wireless sensor network nodes. The simulation results is evidenced with the hyperheuristic energy model in comparison with the state-of-the-art benchmarked clustering algorithms. The energy efficiency management adds heterogeneity in the network by introducing minimal cluster head packets to conserve energy more superior to the DEEC_E, DDEEC_E, and DEEC. Therefore, the simulation reveals that the novel Gaussian algorithm has better optimized performance, and it helps to extend the network lifetime, produce competitive network throughput execution time to the base station, and optimize energy efficiency and stability of network to overcome the challenges of well recognised algorithms. Then, we tackled the maximization problem of network life by integrating a tactic for choosing a representative from each cluster that will allow more energy since it will control the other nodes. The proposed DEEC_GAUSS elimination clustering algorithm has an optimal duration to send packets successfully to the base station (BS). In conclusion we can say that the proposed DEEC_GAUSS algorithm provides an optimized energy-efficient clustering to ensure a more stabilized period for WSN flow of operation. Our recommendation for the future will be to apply the proposed algorithms to thousands of sensor nodes with varieties of energy require- ments to evaluate their efficiency and to overcome any challenges that might be required. The future scope of this work will cover the simulation of the improved proposed algorithm on larger sensor nodes. Furthermore, node localization problem will be addressed for specific applications.

\section{Data Availability}

All data is available from the authors.

\section{Conflicts of Interest}

The authors declare no conflict of interest.

\section{Acknowledgments}

This research was funded by the ICT and Society Research Group, Information Systems, Durban University of Technology.

\section{References}

[1] C. C. Ghosh and H. Moustafa, Localization in machine-tomachine systems, vol. 10, Patent and Trademark Office, Washington, DC: U.S, 2019.

[2] J. Y. Khan and M. R. E. Yuce, Internet of Things (IoT): Systems and Applications, CRC Press, 2019.

[3] R. Sharma, "Enhancement of relay nodes communication approach in WSN-IoT for underground coal mine," Journal of Information and Optimization Sciences, vol. 41, no. 2, pp. 521-531, 2020.

[4] A. Pal, "Localization algorithms in wireless sensor networks: current approaches and future challenges," Network Protocols and Algorithms, vol. 2, pp. 45-73, 2010.

[5] M. Carlos-Mancilla, E. López-Mellado, and M. Siller, "Wireless sensor networks formation: approaches and techniques," Journal of Sensors, vol. 2016, 18 pages, 2016.

[6] N. Jamal, A.-K. Ahmed, and E. Kamal, "Routing techniques in wireless sensor networks: a survey," IEEE wireless communications, vol. 11, no. 6, pp. 26-28, 2004.

[7] T. Pino, S. Choudhury, and F. Al-Turjman, "Dominating set algorithms for wireless sensor networks survivability," IETE Access, vol. 6, pp. 17527-17532, 2018.

[8] K. C. Lan and M. Z. Wei, "A compressibility-based clustering algorithm for hierarchical compressive data gathering," IEEE Sensors Journal, vol. 17, no. 8, pp. 2550-2562, 2017.

[9] P. Kale and M. J. Nene, "Path reestablishment in wireless sensor networks," in process of International Conference on Wireless Communications, Signal Processing and Networking (WiSPNET), pp. 1659-1663, Chennai, 2017.

[10] R. Sharma and S. Malhotra, "Approximate point in triangulation (apit) based localization algorithm in wireless sensor network," International Journal for Innovative Research in Science \& Technology, vol. 2, pp. 39-42, 2015.

[11] E. Shakshuki, A. A. Elkhail, I. Nemer, M. Adam, and T. Sheltami, "Comparative study on range-free localization algorithms," Procedia Computer Science, vol. 151, pp. 501510, 2019.

[12] S. Singh, S. Kumar, A. Nayyar, F. Al-Turjman, and L. Mostarda, "Proficient QoS-based target coverage problem 
in wireless sensor networks," IEEE Access, vol. 8, pp. 7431574325, 2020.

[13] V. K. Menaria, S. C. Jain, N. Raju, R. Kumari, A. Nayyar, and E. Hosain, "NLFFT: a novel fault tolerance model using artificial intelligence to improve performance in wireless sensor networks," IEEE Access, vol. 8, pp. 149231-149254, 2020.

[14] J. Hudson, J. Denzinger, H. Kasinger, and B. Bauer, "Dependable risk-aware efficiency improvement for self-organizing emergent systems," in IEEE Fifth International Conference on Self-Adaptive and Self-Organizing Systems, pp. 11-20, Ann Arbor, MI, USA, 2011.

[15] P. Cowling, G. Kendall, and E. Soubeiga, "A hyperheuristic approach for scheduling a sales summit," in Selected Papers of the Third International Conference on the Practice And Theory of Automated Timetabling, PATAT 2000, Lecture Notes in Computer Science, Springer Journal, pp. 176-190, Berlin, 2002.

[16] R. Qu and E. K. Burke, "Hybridisations within a graph-based hyper-heuristic framework for university timetabling problems," Journal of the Operational Research Society, vol. 60, pp. 1273-1285, 2019.

[17] E. K. Burke, M. R. Hyde, G. Kendall, and G. Woodward, “A genetic programming hyper-heuristic approach for evolving two-dimensional strip packing heuristics," IEEE Transactions on Evolutionary Computation, 2010.

[18] E. K. Burke, E. Hart, G. Kendall, J. Newall, and P. Ross, "Hyper-heuristics: an emerging direction in modern search technology," in Handbook of metaheuristics, F. Glover and G. Kochenberger, Eds., pp. 457-474, Springer, Boston, MA, 2003.

[19] E. K. Burke, S. Petrovic, and R. Qu, "Case-based heuristic selection for timetabling problems," Journal of Scheduling, vol. 9, no. 2, pp. 115-132, 2006.

[20] O. J. Aroba, N. Naicker, A. T. Timothy, and E. Ogunsakin, "Meta-analysis of heuristic approaches for optimizing node localization and energy efficiency in wireless sensor networks," International Journal of Engineering and Advanced Technology (IJEAT), vol. 10, no. 1, pp. 73-87, 2020.

[21] A. A. Babayo, H. M. Anisi, and I. Ali, "A review on energy management scheme in energy harvesting wireless sensor network," Renewable and Sustainable Energy Reviews, vol. 76, pp. 1176-1184, 2017.

[22] R. Krishnamurthi, A. Kumar, D. Gopinathan, A. Nayyar, and B. Qureshi, "An overview of IoT sensor data processing, fusion, and analysis techniques," Sensors, vol. 20, no. 21, p. 6076, 2020.

[23] S. R. Sujatha and M. Siddappa, "Node localization method for wireless sensor networks based on hybrid optimization of particle swarm optimization and differential evolution," IOSR Journal of Computer Engineering, vol. 19, no. 2, pp. 7-12, 2017.

[24] S. R. Sujatha and M. Siddappa, "Self-adaptive differential evolution-based localization of sensors in the wireless sensor network," International Journal of Applied Engineering Research, vol. 13, pp. 86-90, 2018.

[25] L. Cui, C. Xu, G. Li, Z. Ming, Y. Feng, and N. Lu, "A high accurate localization algorithm with DV-Hop and differential evolution for wireless sensor network," Applied Soft Computing, vol. 68, pp. 39-52, 2018.

[26] A. Cespedes-Mota, G. Castanon, A. F. Martinez-Herrera, L. E. Cardenas-Barron, and A. M. Sarmiento, "Differential evolution algorithm applied to wireless sensor distribution on different geometric shapes with area and energy optimization,"
Journal of Network and Computer Applications, vol. 119, pp. 14-23, 2018.

[27] Q. Wan, M. J. Weng, and S. Liu, “Optimization of wireless sensor networks based on differential evolution algorithm," International Journal of Online \& Biomedical Engineering, vol. 15, pp. 183-195, 2019.

[28] Q. Yan, W. Peng, and G. Zhang, "Optimal energy consumption tasks scheduling strategy for multi-radio WSNs," Sensors, vol. 20, no. 3, p. 881, 2020.

[29] X. Yuan, M. Elhoseny, H. K. El-Minir, and A. M. Riad, "A genetic algorithm-based, dynamic clustering method towards improved WSN longevity," Journal of Network and Systems Management, vol. 25, no. 1, pp. 21-46, 2017.

[30] Z. J. Wang, Z. H. Zhan, and J. Zhang, "Solving the energyefficient coverage problem in wireless sensor networks. A distributed genetic algorithm approach with a hierarchical fitness evaluation," Energies, vol. 11, no. 12, p. 3526, 2018.

[31] R. Hamidouche, Z. Aliouat, and A. M. Gueroui, "Genetic algorithm for improving the lifetime and QoS of wireless sensor networks," Wireless Personal Communications, vol. 101, pp. 2313-2348, 2018.

[32] T. S. Panag and J. S. Dhillon, “A novel random transition based PSO algorithm to maximize the lifetime of wireless sensor networks," Wireless Personal Communications, vol. 98, pp. 22612290, 2018.

[33] S. P. Singh and S. C. Sharma, "Implementation of a PSO based improved localization algorithm for wireless sensor networks," IETE Journal of Research, vol. 65, pp. 502-514, 2019.

[34] R. M. Garimella, D. R. Edla, and V. Kuppili, Energy Efficient Design of Wireless Sensor Network: Clustering, International Institute of Information Technology, 2018.

[35] R. Thiagarajan and R. Moorthi, "Energy consumption and network connectivity based on novel-LEACH-POS protocol networks," Computer Communications, vol. 149, pp. 90-98, 2020.

[36] K. A. Darabkh, K. A. Darabkh, N. J. Al-Maaitah, I. F. Jafar, and A.'. F. Khalifeh, "Energy efficient clustering algorithm for wireless sensor networks," in 2017 International Conference on Wireless Communications, Signal Processing and Networking (WiSPNET), Chennai, India, 2017.

[37] S. Shivapur, S. G. Kanakaraddi, and A. K. Chikaraddi, "Load balancing techniques in wireless sensor networks: a comparative study," International Journal of Emerging Technology in Computer Science and Electronics, vol. 14, no. 2, pp. 1-6, 2015.

[38] F. Kiani and A. Seyyedabbasi, "Wireless sensor network and internet of things in precision agriculture," International Journal of Advanced Computer Science and Applications (IJASCA), vol. 9, no. 6, 2018.

[39] J. G. Li, "Optimal routing with scheduling and channel assignment in multi-power multi-radio wireless sensor networks," Ad Hoc Network, vol. 31, pp. 45-62, 2015.

[40] M. Elshrkawey, S. M. Elsherif, and M. E. Wahed, "An enhancement approach for reducing energy consumption in wireless sensor networks," Journal of King Saud University-Computer and Information Sciences, vol. 30, pp. 259-267, 2018.

[41] J. Roselin, P. Latha, and S. Benitta, "Maximizing the wireless sensor networks lifetime through energy-efficient connected coverage," Ad Hoc Network, vol. 62, pp. 1-10, 2017.

[42] J. Zhang, J. Yin, T. Xu, Z. Gao, H. Qi, and H. Yin, “The optimal game model of energy consumption for nodes cooperation in 
WSN," Journal of Ambient Intelligence and Humanized Computing, vol. 11, pp. 589-599, 2018.

[43] J. Kennedy and R. C. Eberhart, "Particle swarm optimization," in Proceedings of IEEE international conference on neural networks, vol. 27, pp. 1942-1948, Piscataway, NJ, 2019.

[44] S. Pang, F. Wang, Z. Zhen et al., "Pattern classification and PSO optimal weights based sky images cloud motion speed calculation method for solar PV power forecasting," IEEE Transactions on Industry Applications, vol. 55, pp. 33313342, 2018.

[45] J. Wang, Y. Gao, W. Liu, A. K. Sangajath, and H. J. Kim, “An improved routing schema with special clustering using PSO algorithm for heterogeneous wireless sensor network," Sensors, vol. 19, no. 3, p. 671, 2019.

[46] B. M. Sahoo, A. D. Gupta, S. A. Yadav, and S. Gupta, "ESRA: enhanced stable routing algorithm for heterogeneous wireless sensor networks," in International Conference on Automation, Computational and Technology Management (ICACTM), pp. 148-152, London, United Kingdom, United Kingdom, 2019.

[47] T. A. Al-Janabi and H. S. Al-Raweshidy, "Optimised clustering algorithm-based centralised architecture for load balancing in IoT network," in 2017 International Symposium on Wireless Communication Systems (ISWCS), pp. 269-274, Bologna, Italy, 2017.

[48] W. B. Heinzelman, A. P. Chandrakasan, and H. Balakrishnan, "An application-specific protocol architecture for wireless microsensor networks," IEEE Transactions on Wireless Communications, vol. 1, no. 4, pp. 660-670, 2002.

[49] S. Gharib, S. R. Ali, R. Khan, and N. Munir, "System of Linear Equations, Guassian Elimination," Global Journal of Computer Science and Technology, vol. 15, no. 5-C, 2015.

[50] L. de Oliveira Miranda and L. B. B. Miranda, "Lohans' magic squares and the Gaussian elimination method," Journal of Nepal Mathematical Society, vol. 3, no. 1, pp. 31-36, 2020. 